\title{
Naturwissenschaft pur? - Zu den Ambitionen und Erfolgsaussichten einer naturalistischen Metaphysik
}

Matthias Egg

Was ist das Ziel der Philosophie? Wilfrid Sellars beantwortet in einem einflussreichen Aufsatz die Frage so: »to understand how things in the broadest possible sense of the term hang together in the broadest possible sense of the term « ${ }^{1}$. Im weiteren Verlauf seines Aufsatzes führt Sellars diesen Gedanken dahingehend aus, dass eine zentrale Aufgabe der Philosophie darin bestehe, das naturwissenschaftliche ${ }^{2}$ Weltbild mit dem, was er das "manifeste« (in gewissem Sinne vortheoretische) Weltbild nennt, in Einklang zu bringen.

Ohne näher auf Sellars' Beschreibung dieser beiden Weltbilder einzugehen, drängen sich sogleich zwei Bemerkungen auf: Erstens sollte man sich angesichts der kaum überschaubaren Vielfalt der heute verfügbaren wissenschaftlichen Erkenntnisse bewusst sein, dass schon die kohärente Artikulation "des" naturwissenschaftlichen Weltbildes eine riesige philosophische Leistung (im eingangs erwähnten Sinn des »Verstehens, wie die Dinge zusammenhängen«) erfordert. Zweitens hat die fortschreitende Erweiterung naturwissenschaftlicher Erkenntnisgebiete zur weit verbreiteten philosophischen Ansicht geführt, dass mit dem Erbringen dieser Leistung die ganze von Sellars skizzierte Aufgabe schon erfüllt ist: Das naturwissenschaftliche Bild, so die Ansicht, wird dereinst die ganze Wirklichkeit abbilden, so dass die geforderte Vereinbarung mit dem manifesten Bild dann schlicht darin besteht, letzteres durch ersteres zu ersetzen. In der gegenwärtigen philosophischen Debatte äussert sich diese Ansicht prominent im Rahmen der sogenannten naturalistischen (oder naturwissenschaftlichen) Metaphysik, mit der ich mich im vorliegenden Aufsatz kritisch auseinandersetze.

Dass es überhaupt so etwas wie naturwissenschaftliche Metaphysik geben soll, mag erstaunen, wurde doch der Begriff der Metaphysik

\footnotetext{
1 Wilfrid Sellars, Philosophy and the Scientific Image of Man [1962], in: Ders., Empiricism and the Philosophy of Mind, London 1963,1-40, hier 1.

2 Bekanntlich hat der (von Sellars verwendete) Begriff "scientific" im Deutschen keine exakte Entsprechung. "wissenschaftlich" wäre zu weit gefasst, da die Geisteswissenschaften keine "sciences" sind. Die hier gewählte Übersetzung "naturwissenschaftlich « ist eigentlich zu eng, da sie die empirischen Sozialwissenschaften (social sciences) nicht berücksichtigt. Für den gegenwärtigen Kontext ist dies aber nicht gravierend.
} 
in seiner wechselvollen Geschichte oft dem Begriff der empirischen Wissenschaft entgegengesetzt. So spricht etwa der klassische Positivismus nach Auguste Comte von einer "metaphysischen Epoche" in der Entwicklungsgeschichte der Menschheit, die von der "positiven" (d.h. empirisch-wissenschaftlichen) Epoche abgelöst werden soll, nachdem sie zuvor ihrerseits die "theologische Epoche« abgelöst hat. Das Erbe dieses Gedankens findet sich im Neopositivismus des frühen 20. Jahrhunderts in der Vorstellung, dass Metaphysik durch wissenschaftlich-analytisches Denken überwunden werden kann und soll. Mit dem Niedergang des logischen Empirismus in der zweiten Hälfte des 20. Jahrhunderts ist aber die Metaphysik auch innerhalb der sogenannt analytischen Philosophie wieder zu einer akzeptierten Disziplin geworden, so dass heute viele Philosophinnen und Philosophen nicht mehr in Frage stellen, dass man Metaphysik betreiben soll, sich jedoch hitzige Debatten darüber liefern, wie man es tun soll.

Bei aller historischen Fragwürdigkeit scheint mir Comtes Charakterisierung der Metaphysik als Übergangsphase (und damit gleichsam als Bindeglied) zwischen einer theologisch-religiös und einer empirisch-wissenschaftlich geprägten Epoche in systematischer Hinsicht äusserst aufschlussreich. Wie ich im Folgenden zeigen werde, verbindet sich auch heute noch mit dem Projekt der naturalistischen Metaphysik ein zumindest quasi-religiöser Anspruch, der sich im Wunsch nach einer Lebens-orientierenden Funktion wissenschaftlicher Erkenntnisse manifestiert. Im Anschluss daran werde ich die Fragen behandeln, inwiefern Metaphysik ein notwendiges, und inwiefern sie ein erfolgversprechendes Mittel zur Erfüllung dieses Wunsches ist.

Betrachten wir zunächst, wie zwei der prominentesten Verfechter der naturalistischen Metaphysik, James Ladyman und Don Ross, ihre eigene Motivation für dieses Projekt beschreiben:

"The best motivation for trying to synthesize our scientific knowledge into a unified picture - that is, for building naturalistic metaphysics - is the crucial service this activity potentially performs in extending the Enlightenment project. If science is not seen to provide the basis for a general worldview, then people will continue to collectively confabulate alternative general pictures. This in turn matters because the confabulated pictures 
inspire groundless and usually wasteful and destructive politics and policy. $\star^{3}$

Der aufklärerische Anspruch, die Menschen von ihren unbegründeten, verschwenderischen und zerstörerischen Handlungsweisen abzubringen, kann also gemäss Ladyman und Ross nicht von den Wissenschaften allein eingelöst werden, es braucht zusätzlich die Synthese wissenschaftlicher Erkenntnisse im Rahmen einer naturalistischen Metaphysik. Damit kann nicht gemeint sein, dass wissenschaftliche Erkenntnisse für sich allein niemals handlungsleitend sein können; niemand würde behaupten, dass konkrete Forschungsergebnisse etwa aus den Ingenieurswissenschaften, der Agronomie oder der Medizin nur dann praxisrelevant werden, wenn sie sich in ein metaphysisches Gesamtsystem integrieren lassen. Was Ladyman und Ross im Blick haben, sind vielmehr grundlegende gesellschaftliche, moralische und existenzielle Fragen, und hier ist es tatsächlich plausibel, dass die Wissenschaften allein nicht die Kraft haben, das menschliche Handeln zu orientieren. Womöglich braucht es dazu umfassende Weltbilder von der Art, wie sie traditionellerweise von Religionen geliefert werden.

Nun ist die Klage über die mangelnde existenzielle Relevanz wissenschaftlicher Erkenntnisse keineswegs neu. Bereits kurz vor dem zweiten Weltkrieg diagnostizierte Edmund Husserl eine »Krisis der europäischen Wissenschaften«, die er dem »Verlust ihrer Lebensbedeutsamkeit« zuschrieb. Das damalige Unbehagen schildert er in dramatischen Worten:

"In unserer Lebensnot - so hören wir - hat diese Wissenschaft uns nichts zu sagen. Gerade die Fragen schliesst sie prinzipiell aus, die für den in unseren unseligen Zeiten den schicksalsvollsten Umwälzungen preisgegebenen Menschen die brennenden sind: die Fragen nach Sinn und Sinnlosigkeit dieses ganzen menschlichen Daseins. $\ll^{4}$

Husserl hätte kaum Sympathie für den Vorschlag aufgebracht, den Ausweg aus dieser Situation in einer naturalistischen Metaphysik à la Ladyman/Ross zu suchen. Stattdessen sieht er in der von ihm entwickelten transzendentalen Phänomenologie (verstanden als eine "Wissenschaft von der Lebenswelt») das Potenzial, der Wissenschaft ihre Lebensbedeutsamkeit zurückzugeben. Allerdings gibt es, wie

\footnotetext{
3 James Ladyman/Don Ross, The world in the data, in: Scientific Metaphysics, hg. v. Don Ross/James Ladyman/Harold Kincaid, Oxford 2013, 108-150, hier 113.

4 Edmund Husserl, Die Krisis der europäischen Wissenschaften und die transzendentale Phänomenologie [1936], Den Haag ${ }^{2} 1976,4$.
} 
Jürgen Habermas ausführt, Grund zur Annahme, dass Husserl selbst implizit auf ein metaphysisches (oder wie Habermas es auch nennt, kosmologisches) Verständnis wissenschaftlicher Resultate zurückgreift, wenn er von ihnen Lebensbedeutsamkeit erwartet. Ohne diese kosmologische Komponente wäre nämlich, so Habermas, nicht einzusehen, wie die der Phänomenologie zugrunde liegende theoretische Einstellung eine praktisch wirksame Wissenschaft hervorbringen soll:

"Theorie im Sinne der grossen Tradition ging darum ins Leben über, weil sie in der kosmischen Ordnung einen idealen $\mathrm{Zu}-$ sammenhang der Welt, und das hiess: auch den Prototyp für die Ordnung der Menschenwelt, zu entdecken vermeinte. Nur als Kosmologie war Theoria zugleich der Orientierung des Handelns mächtig. $\|^{5}$

Wenn Habermas' Kritik zutrifft, dann liegt Husserls Wissenschaftsverständnis näher bei demjenigen der naturalistischen Metaphysik, als es zunächst aussieht. ${ }^{6}$ Letzterer könnte man dann zugute halten, dass sie die bei Husserl zwar implizit vorhandenen, aber nicht eingestandenen metaphysischen Elemente explizit vertritt, ohne sich dafür zu schämen. Allerdings sind gewisse Zweifel an diesem Zwischenfazit angebracht: Erstens macht es sich Habermas möglicherweise zu einfach, wenn er unterstellt, dass Kosmologie (bzw. Metaphysik) Husserls einzige Möglichkeit ist, einer phänomenologisch erneuerten Wissenschaft Lebensbedeutsamkeit abzuringen. Zweitens wäre es, zumindest aus Habermas' Sicht (für die er später die Bezeichnung "nachmetaphysisches Denken" geprägt hat), auch ganz aussichtslos, von irgendeiner Metaphysik (sei sie nun eingestanden oder nicht) Lebensbedeutsamkeit zu erwarten. Und drittens unterliegt sogar bei Ladyman und Ross selber das Vertrauen in die transformative Kraft der Metaphysik einer gewichtigen Einschränkung, die nun zu thematisieren ist.

In der eingangs skizzierten Geschichte der Metaphysik habe ich erwähnt, dass der Niedergang des logischen Empirismus den Weg für die heutige analytische Metaphysik freigemacht hat. Dies heisst natürlich nicht, dass es nicht auch in jüngerer Zeit Grundsatzkritik

\footnotetext{
5 Jürgen Habermas, Erkenntnis und Interesse [1965], in: Ders., Technik und Wissenschaft als >Ideologie‘, Frankfurt 1968, 146-168, hier 152-153.

6 Dies führe ich in meinem Beitrag "A revealing parallel between Husserl's philosophy of science and today's scientific metaphysics" für den Band "Phenomenological Approaches to Physics" (hg. v. Harald Wiltsche und Philipp Berghofer, Synthese Library, im Erscheinen) weiter aus.
} 
an dieser Art des Philosophierens gegeben hätte. Ladyman und Ross nehmen diese Kritik auf, was sie zu einer Unterscheidung zwischen starker Metaphysik (die sie selber auch ablehnen) und schwacher Metaphysik (die sie gutheissen) führt. Im Unterschied zur starken Metaphysik behandelt die schwache Variante philosophische Positionen nicht als Doktrinen, sondern als Haltungen oder Einstellungen (engl. "stances") im Sinn von Bas van Fraassen. ${ }^{7}$ Schwache Metaphysik besteht also nicht (oder nicht in erster Linie) darin, irgendwelche Behauptungen über die Wirklichkeit aufzustellen und zu verteidigen, sondern auf der Basis einer bestimmten Einstellung ein kohärentes Weltbild zu artikulieren. Die Unterscheidung zwischen starker und schwacher Metaphysik ist nicht unproblematisch, wie man leicht einsieht, sobald man die Frage stellt, was denn nun der Status der Aussagen ist, die sich aus dem in der schwachen Metaphysik enthaltenen Artikulationsprozess ergeben. Offenbar dürfen sie keinen doktrinären Charakter haben, denn damit würde man in der starken Metaphysik landen, und es ist nicht ganz klar, wie dies verhindert werden soll. Ich brauche dieses Problem aber hier nicht in seiner vollen Allgemeinheit zu behandeln, sondern kann mich auf den Gebrauch beschränken, den Ladyman und Ross von der Unterscheidung zwischen starker und schwacher Metaphysik machen.

Ladyman und Ross selber bezeichnen die Haltung, die sie zur Grundlage ihrer (schwachen) Metaphysik machen, als "szientistisch" und beschreiben sie als eine Kombination einer empiristischen und einer materialistischen Haltung. ${ }^{8}$ Ich werde diese Kombination sogleich genauer untersuchen, möchte zunächst aber noch bei der Frage bleiben, was nun starke und schwache Metaphysik voneinander unterscheidet. Aufschluss darüber gibt Ladymans und Ross' Beschreibung ihres Umgangs mit Gesprächspartnern, die ihre szientistische Haltung nicht teilen:

"Their resistance to science, which must be quite thoroughgoing if it is not to be unprincipled, will confront them with serious policy problems in the management of social affairs, and we will want to press them as hard as possible on these. But we would not try to convert them with metaphysics, for van Fraassen is right that that would require strong metaphysics, and strong metaphysics can't get off the ground. «"

\footnotetext{
7 Bas C. van Fraassen, The Empirical Stance, Yale 2002.

8 Vgl. James Ladyman, The scientistic stance: the empirical and materialist stances reconciled, in: Synthese 178 (2011), 87-98.

9 James Ladyman und Don Ross, Every Thing Must Go. Metaphysics Naturalized, Oxford 2007, 64.
} 
Der hier behauptete Unwille, jemanden "mit Metaphysik zu bekehren«, passt offensichtlich nicht so recht zur weiter oben zitierten Motivation für naturalistische Metaphysik. Denn obwohl das dort beschriebene Vorhaben nur schwache Metaphysik (d.h. die kohärente Artikulation eines szientistischen Weltbilds) erforderte, so bestand das Ziel doch explizit darin, Leute von ihren »konfabulierten alternativen Weltbildern« abzubringen. Und dabei spielte (schwache) Metaphysik doch zumindest die Rolle, das szientistische Weltbild wenn nicht als wahr (denn das wäre starke Metaphysik) so doch als kohärent und somit als attraktiv darzustellen.

Es ist übrigens kein Zufall, dass in diesem Zusammenhang der religiös konnotierte Begriff der »Bekehrung« auftritt (wobei anzumerken ist, dass das im Englischen verwendete Wort "conversion« etwas weniger eindeutig religiös konnotiert ist als "Bekehrung«). Dieser spielt bereits bei van Fraassen eine zentrale Rolle, wenn er in The Empirical Stance die Idee der philosophischen »Haltung« entwickelt, die Ladymans und Ross' Konzept der schwachen Metaphysik zugrunde liegt. Diese Idee dient van Fraassen nämlich unter anderem dazu, die radikalen, scheinbar nicht rein rational begründbaren Veränderungen des Denkens verständlich zu machen, die Thomas S. Kuhn als "wissenschaftliche Revolutionen« (und auf der individuellen Ebene selbst schon als "Bekehrungen«) bezeichnet hat. ${ }^{10}$

Der gleichsam religiöse Charakter philosophischer Haltungen tritt aber nicht nur im historischen Prozess ihrer gegenseitigen Ablösung zutage, sondern auch in der systematischen Beschreibung konkreter solcher Haltungen, wie nun am Beispiel der von Ladyman und Ross propagierten szientistischen Haltung zu sehen sein wird. Wie oben erwähnt, setzt diese sich aus einer empiristischen und einer materialistischen (oder vielleicht besser: physikalistischen ${ }^{11}$ ) Haltung zusammen. Die Pointe dieser Kombination besteht darin, dass van Fraassen diese beiden Komponenten als Gegensätze verstanden hat, da der Materialismus (den van Fraassen ablehnt) zu metaphysischen Aussagen neigt, die der anti-metaphysischen Einstellung des Empirismus (den er vertritt) zuwiderlaufen. Ladyman versucht nun, die scheinbar gegensätzlichen Haltungen miteinander zu versöhnen: Einerseits teilt er mit van Fraassen die empiristische Ablehnung der

${ }^{10}$ Vgl. Van Fraassen, Empirical Stance, Kap. 3; Thomas S. Kuhn, The Structure of Scientific Revolutions [1962], Chicago ${ }^{2} 1970$.

${ }^{11}$ Van Fraassen verwendet nicht nur "Materialismus" und "Physikalismus«, sondern auch "Naturalismus" mit (für seine Zwecke) gleicher Bedeutung (Empirical Stance, 49), und Ladyman/Ross übernehmen (zumindest für den Kontext ihrer Auseinandersetzung mit van Fraassen) diesen Sprachgebrauch (Every Thing Must Go, 62-63; Scientistic Stance, 91). 
herkömmlichen (nicht-naturalistischen) Metaphysik ${ }^{12}$, andererseits hält er aber eine rein empiristische Haltung für defizitär, weil sie erstens die Rolle der Metaphysik als Inspirationsquelle für weitere empirische Forschung übersieht ${ }^{13}$ und zweitens den modalen Gehalt wissenschaftlicher Theorien (also ihre Aussagen nicht nur über das, was wirklich, sondern auch über das, was möglich bzw. unmöglich ist) nicht adäquat berücksichtigen kann. ${ }^{14}$ Im Hintergrund steht dabei wiederum der bereits oben angesprochene, quasi-religiöse Impuls, die einzelnen wissenschaftlichen Erkenntnisse nicht einfach (in empiristischer Manier) nebeneinander stehen zu lassen, sondern sie zu einem umfassenden und (so hofft man) kohärenten Gesamtbild $\mathrm{zu}$ verweben, das dem Menschen Orientierung zu geben vermag.

Die Rede von einem quasi-religiösen Impuls innerhalb der szientistischen Haltung mag fragwürdig erscheinen, da insbesondere Ladyman explizit den säkularen Charakter seiner Haltung betont. ${ }^{15}$ Zweifellos besteht ein solcher Zusammenhang zwischen Szientismus und Säkularismus, es fragt sich jedoch, auf welcher der beiden Komponenten der szientistischen Haltung dieser Zusammenhang beruht. Im Gegensatz zu Ladyman sieht van Fraassen etwa keinen notwendigen Zusammenhang zwischen Empirismus und Säkularismus, da letzterer eine Vollständigkeit des naturwissenschaftlichen Weltbilds postuliert, die der Empirismus, wie van Fraassen ihn versteht, nicht annehmen muss. Dies ermöglicht es van Fraassen, seinen Empirismus mit einer dezidiert nicht-säkularen Haltung zu kombinieren. ${ }^{16}$ Der springende Punkt ist nun, dass auch Empiristen, die dies nicht tun, den Wunsch nach Einbettung in eine umfassende, Lebens-orientierende Erzählung haben können. Dies ist es, was ich den quasi-religiösen Impuls nenne, der Ladyman und Ross dazu veranlasst, neben der empiristischen auch die materialistische Haltung einzunehmen und auf dieser Grundlage ihre naturalistische Metaphysik zu entwickeln.

Gegen die Erfolgsaussichten dieses Projekts sind verschiedene Einwände erhoben worden. Ein durchaus gewichtiger, den ich aber hier nicht weiter diskutiere, besteht darin, dass bisherige Versuche, für unser Selbstverständnis zentrale Kategorien wie Bewusstsein, Vernunft oder Moral in ein rein naturalistisches Weltbild zu inte-

\footnotetext{
12 Vgl. Ladyman, Scientistic Stance, 89.

3 Vgl. ebd., 93.

${ }^{14}$ Vgl. ebd., 94-95.

15 Vgl. ebd., 97.

${ }^{16}$ Vgl.Van Fraassen, Empirical Stance, Kap. 5.
} 
grieren, wenig überzeugend sind. ${ }^{17}$ Ein weiteres Problem ist die Unmöglichkeit, eine klare Grenze zu ziehen zwischen derjenigen Metaphysik, die aus naturalistischer Sicht unbedenklich ist (weil sie auf den Resultaten empirischer Wissenschaften gründet) und derjenigen Metaphysik, die sich in ihren Spekulationen zu weit von der empirischen Forschung abgekoppelt hat. ${ }^{18}$ Dieses Problem erscheint vielleicht nicht allzu gravierend, da auch eine Unterscheidung mit unscharfer Grenze eine substanzielle Unterscheidung sein kann. Es zeigt sich aber, dass in gewissen Teilgebieten der Wissenschaft die Spannung zwischen den von der empirischen Forschung nahegelegten metaphysischen Postulaten und der dem Naturalismus innewohnenden Metaphysik-Skepsis so gross wird, dass sie selbst die Kohärenz einer naturalistischen Metaphysik bedroht. Das wohl drastischste Beispiel hierfür liefert die Quantenmechanik. Die folgende, sehr knappe Darstellung dieses Beispiels soll lediglich einen Eindruck der Herausforderung vermitteln, denen sich das Projekt der naturalistischen Metaphysik nur schon im Kontext der Physik gegenübersieht. ${ }^{19}$

Die Quantenmechanik ist eine der empirisch erfolgreichsten, gleichzeitig aber auch am wenigsten gut verstandenen wissenschaftlichen Theorien überhaupt. Ein wesentlicher Teil ihres empirischen Erfolgs beruht auf der sogenannten Bornschen Regel, die es erlaubt, aus dem theoretischen Formalismus präzise (und hervorragend bestätigte) Vorhersagen über die Wahrscheinlichkeiten von Ergebnissen bestimmter Messungen abzuleiten. So lässt sich mit Hilfe der Bornschen Regel beispielsweise die Wahrscheinlichkeit berechnen, dass ein von einer radioaktiven Quelle ausgesandtes Teilchen auf einer bestimmten Stelle eines fluoreszierenden Schirms auftrifft. Die vielfach wiederholte Durchführung des entsprechenden Experiments bestätigt dann mit hoher Genauigkeit, dass die von der Quantenmechanik vorhergesagten Wahrscheinlichkeiten tatsächlich zutreffen.

Wie der Name schon sagt, ist die Bornsche Regel etwas, dem man folgen kann, um im Experimentieren Erfolg zu haben, sie wird aber gewöhnlich nicht als (Natur-) Gesetz verstanden, das uns über funda-

\footnotetext{
17 Vgl. z.B. Thomas Nagel, Mind and Cosmos: Why the Materialist Neo-Darwinian Conception of Nature is Almost Certainly False, Oxford 2012.

${ }_{18}$ Vgl. Anjan Chakravartty, Scientific Ontology. Integrating Naturalized Metaphysics and Voluntarist Epistemology, Oxford 2017, Kap. 3. Kernthese dieses Kapitels: "There is no objective distinction between theorizing and speculating in the context of scientific ontology" (89).

${ }^{19}$ Für eine ausführlichere Darstellung vgl. Matthias Egg, Dissolving the measurement problem is not an option for the realist, in: Studies in the History and Philosophy of Modern Physics (online publiziert 2018), DOI 10.1016/j.shpsb:2018.12.002.
} 
mentale physikalische Zusammenhänge Auskunft geben würde. Der Grund dafür ist, dass der in ihr vorkommende Begriff der Messung kein physikalischer Grundbegriff sein kann, denn Messungen werden gemacht, sie treten nicht einfach "von Natur aus" auf. ${ }^{20}$

Nun kann man natürlich versuchen, den Messbegriff in rein physikalischen Begriffen zu analysieren, dabei stellt sich aber heraus, dass man auf die eine oder andere Art über das hinausgehen muss, was im herkömmlichen Formalismus der Quantenmechanik enthalten ist. Es gibt verschiedene, sich gegenseitig radikal widersprechende Vorschläge, wie dies zu tun ist. Da aber all diese Vorschläge so konstruiert sind, dass ihre empirischen Vorhersagen mit denjenigen der herkömmlichen Quantenmechanik (zumindest näherungsweise) übereinstimmen, ist es nicht möglich, mit Hilfe von Experimenten $\mathrm{zu}$ entscheiden, welcher von ihnen "der richtige " ist. Somit sind sämtliche Versuche, die Bornsche Regel auf eine solide theoretische Grundlage zu stellen, auf unvermeidliche Weise metaphysisch, indem sie etwa zusätzliche ("verborgene «) Variablen, kleine (mit heutigen Mitteln nicht messbare) Modifikationen der dynamischen Gesetze oder die Existenz unbeobachtbarer Parallel-Welten postulieren. ${ }^{21}$

Insofern die genannten Vorschläge durch das Ziel motiviert sind, den empirischen Erfolg der Quantenmechanik theoretisch kohärent zu begründen, kann jeder von ihnen für sich reklamieren, legitimer Teil einer naturalistischen Metaphysik zu sein. Da sie aber miteinander inkompatibel sind und sich keinerlei wissenschaftlicher Konsens darüber abzeichnet, welchem von ihnen nun der Vorzug zu geben sei, ist es aus naturalistischer Sicht kaum gerechtfertigt, sich einfach einem von ihnen anzuschliessen. Dies führt Ladyman und Ross dazu, schon den Ausgangspunkt dieser metaphysischen Projekte in Frage zu stellen, nämlich die angebliche Notwendigkeit einer Analyse des Messbegriffs. In Berufung auf Niels Bohr behaupten sie, dass der

\footnotetext{
${ }^{20}$ Der locus classicus für diese Kritik am Messbegriff ist John S. Bell, Against 'measurement [1990], in: Ders., Speakable and Unspeakable in Quantum Mechanics [1987], Cambridge ${ }^{2} 2004$, Kap. 23.

${ }^{21}$ Für weitere Ausführungen verweise ich auf die umfangreiche Literatur zum Messproblem der Quantenmechanik. Einen guten Überblick mit vielen weiteren Referenzen liefert Wayne Myrvolds Eintrag "Philosophical Issues in Quantum Theory in der Stanford Encyclopedia of Philosophy (https://plato.stanford.edu/archives/spr2017/entries/qt-issues/). Im deutschsprachigen Bereich ist zu empfehlen: Cord Friebe/Meinard Kuhlmann/Holger Lyre/Paul Näger/Oliver Passon/Manfred Stöckler, Philosophie der Quantenphysik. Einführung und Diskussion der zentralen Begriffe und Problemstellungen der Quantentheorie für Physiker und Philosophen, Berlin 2015.
} 
empirische Erfolg der Quantenmechanik auch ohne eine solche Analyse verständlich gemacht werden kann. ${ }^{22}$

Es stellt sich aber heraus, dass damit genau diejenigen Motive unterlaufen werden, die Ladyman und Ross dazu veranlasst haben, sich nicht mit einer rein empiristischen Haltung zufrieden zu geben, sondern sie durch die materialistische Haltung zu ergänzen. Wird nämlich die Bornsche Regel als nicht weiter analysierbares Gesetz verstanden, so gibt man damit die Hoffnung auf ein umfassendes naturwissenschaftliches Weltbild auf, da gewisse Fragen über tatsächlich ablaufende Prozesse aus dem Bereich des wissenschaftlich zulässigen Fragens ausgeschlossen werden. Ein simples Beispiel dafür ist die Frage, ob ein Messgerät auch dann ein Ergebnis anzeigt, wenn niemand hinschaut: Verzichtet man auf eine physikalische Analyse des Messbegriffs, so ist man in einem solchen Fall nicht berechtigt, von einer Messung zu sprechen (denn unser vor-analytischer Messbegriff schliesst zweifellos die Aktivität einer messenden Person mit ein), und somit wird die Anwendung der Bornschen Regel unzulässig. Ohne die Bornsche Regel ist aber die Quantenmechanik ihrer Aussagen über Messergebnisse beraubt.

Wenn meine Beurteilung dieses Falls zutrifft, dann stösst die naturalistische Metaphysik bereits an ihre Grenzen, noch bevor sie sich an diejenigen Wissenschaften gewagt hat, in denen traditionellerweise noch viel grössere Probleme für den Naturalismus diagnostiziert wurden als in der Physik. Ausserdem drängt sich im Licht des zuvor Gesagten die Vermutung auf, dass es die eigenen quasireligiösen Ambitionen sind, an denen die naturalistische Metaphysik letztlich scheitert. Würde sich der Naturalismus strikt an das halten, was die Naturwissenschaften liefern, müsste er sich mit van Fraassens empiristischer Haltung zufriedengeben. Da er aber (zumindest in der Ladyman/Ross'schen Ausführung) nach einem umfassenden Weltbild strebt, handelt er sich das Problem ein, die empiristische mit der materialistischen Haltung in Einklang zu bringen. Das betrachtete Beispiel der Quantenmechanik nährt Zweifel daran, dass diese Versöhnung gelingen kann. Wenn sie aber nicht gelingt, dann ist der naturalistischen Metaphysik, selbst wenn sie bescheiden als schwache Metaphysik verstanden wird, die Grundlage entzogen.

- Dr. Matthias Egg ist Postdoc-Assistent am Institut für Philosophie der Universität Bern. Seine Forschung befasst sich mit Fragen der allgemeinen Wissenschaftsphilosophie sowie der Philosophie der Physik, insbesondere zur Bedeutung fundamentaler physikalischer Theorien.

${ }^{22} \mathrm{Vgl}$. Ladyman/Ross, The world in the data, 134. 